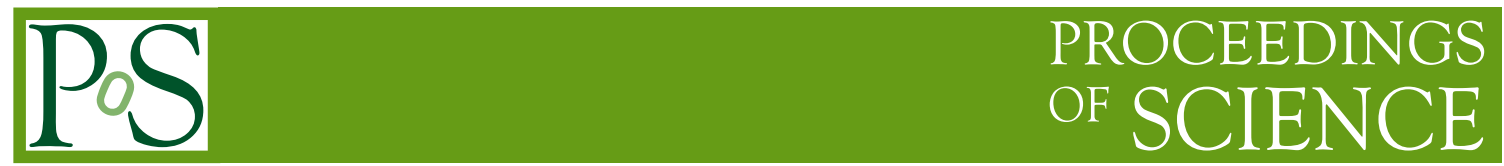

\title{
Analytic results for multiloop scattering amplitudes
}

\author{
J. M. Henn* \\ Institute for Advanced Study, Einstein Drive, Princeton, NJ 08540, USA \\ E-mail: jmhenn@ias.edu
}

The velocity-dependent cusp anomalous dimension is by definition the ultraviolet (UV) anomalous dimension of a Wilson loop with a cusp. It appears in many physically interesting processes. In this talk we present recent progress in the analytic calculation of that quantity.

Loops and Legs in Quantum Field Theory - 11th DESY Workshop on Elementary Particle Physics, April 15-20, 2012

Wernigerode, Germany

${ }^{*}$ Speaker. 


\section{INTRODUCTION}

Wilson loops are very fundamental and important quantities in gauge theories [1]. They are essential for defining nonlocal gauge invariant quantities. They also contain information about local operators via the operator product expansion. Moreover, it turns out that certain Wilson loops defined for specific contours appear as an effective description of certain physical processes.

The velocity-dependent cusp anomalous dimension is a case in point. It is defined as the ultraviolet (UV) anomalous dimension of a Wilson loop with a cusp. It appears in many physically interesting situations. For example, in heavy quark effective theory (HQET), it describes the infrared (IR) divergences of massive form factors and scattering amplitudes, see e.g. [2, 3, 4, 5].

In a $\mathscr{N}=4$ super Yang-Mills (SYM), it is natural to define the locally supersymmetric Wilson loop operator $[6,7]$

$$
W \sim \operatorname{Tr}\left[P \exp \left(i \oint A^{\mu} \dot{x}_{\mu}+\oint|d x| \vec{n} \cdot \vec{\Phi}\right)\right],
$$

where $\vec{n}$ is a vector on $S^{5}$. It parametrizes the coupling of the Wilson loop to the six scalars $\vec{\Phi}$ of the theory. We consider as the integration contour a cusp formed by two segments along directions (momenta) $p^{\mu}$ and $q^{\mu}$, and allow the two segments to couple to the scalars through vectors $\vec{n}$ and $\vec{n}^{\prime}$, see Fig. 1. Then, the vacuum expectation value $\langle W\rangle$ of the Wilson loop will depend on the angles

$$
\cos \phi=\frac{p \cdot q}{\sqrt{p^{2} q^{2}}}, \quad \cos \theta=\vec{n} \cdot \vec{n}^{\prime}
$$

as well as on the 't Hooft coupling $\lambda=g^{2} N$, and the rank of the gauge group $N$.

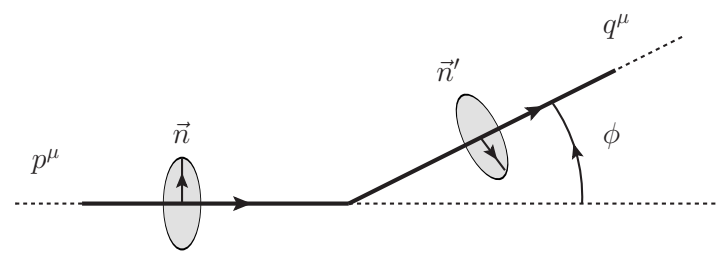

Figure 1: A Wilson line that makes a turn by an angle $\phi$ in Euclidean space. The two segments are directed along $p^{\mu}$ and $q^{\mu}$, respectively. The vectors $\vec{n}$ and $\vec{n}^{\prime}$ are internal vectors that determine the coupling to the scalars $\vec{\Phi}$.

If $\Lambda_{\mathrm{UV}}$ and $\Lambda_{\mathrm{IR}}$ are short and large distance cutoffs, respectively, then the divergent part of the vacuum expectation value of the Wilson loop takes the form $[1,8]$

$$
\langle W\rangle \sim \exp \left[-\log \frac{\Lambda_{\mathrm{UV}}}{\Lambda_{\mathrm{IR}}} \Gamma_{\text {cusp }}+\ldots\right] .
$$

This defines the cusp anomalous dimension $\Gamma_{\text {cusp }}(\phi, \theta, \lambda, N)$.

We will begin by describing the dependence of $\Gamma_{\text {cusp }}$ on the angles $\phi$ and $\theta$ in section 2, and then introduce, in section 3, the set of integral functions needed to express loop-level results. We 
will then review perturbative results to two loops, and present the new three-loop result. We present the light-like limit as a check in section 5, and describe our method of calculation in 6 . We then present an exact result for $\Gamma_{\text {cusp }}$ at small angles in section 7 . In section 8, we discuss a new scaling limit. Finally, we conclude and present future directions in section 9.

\section{KINEMATICS AND LIMITS}

For reasons that will become apparent presently, we will mostly be interested in the $\phi$ dependence of the Wilson loop. It is convenient to introduce a new variable $x=e^{i \phi}$. The computation we are considering is invariant under $\phi \rightarrow-\phi$. This corresponds to an inversion symmetry in $x$.

Note that the dependence of $\Gamma_{\text {cusp }}$ on $\theta$ is simple. It can only occur through Wick contractions of scalars, and because of $\mathrm{SO}(6)$ invariance it appears only through $\vec{n} \cdot \vec{n}^{\prime}=\cos \theta$. Therefore, at $L$ loops, $\Gamma_{\text {cusp }}$ is a polynomial in $\cos \theta$, of maximal degree $L$. Having made this observation, we find that it convenient to introduce the variable $\xi=(\cos \theta-\cos \phi) /(i \sin \phi)$. Two important cases are $\theta=0$ (constant coupling to scalars) and $\theta=\pi / 2$ (scalars on opposite edges are orthogonal to each other), which lead to $\xi=(1-x) /(1+x)$ and $\xi=\left(1+x^{2}\right) /\left(1-x^{2}\right)$, respectively.

There are several special cases of the angles that are of particular interest. When the geometric angle $\phi$ and internal angle $\theta$ satisfy $\phi= \pm \theta$, the anomalous dimension vanishes. Note that $\xi$ vanishes in this case. For $\theta=0$, this corresponds to $\phi \rightarrow 0$, i.e. $x \rightarrow 1$, the case of a straight line. The small angle limit is related to energy loss of an accelerated quark, and is known exactly $[10,11]$. The limit $\phi \rightarrow \pi$, i.e. $x \rightarrow-1$, is related to the quark-antiquark potential. This limit is subtle and requires a resummation of certain diagrams, see [12, 13, 14, 15].

The above limits can be defined in Euclidean space. There is an intrinsically Minkowskian limit that is also of interest. When $\phi \rightarrow-i \varphi, \varphi \gg 1$, i.e. $x \rightarrow 0$, the cusp anomalous dimension diverges linearly in the $\varphi$, to all orders in the coupling constant [9]. The coefficient of the linear divergence is the well-studied light-light cusp anomalous dimension; the latter can also be obtained from the anomalous dimension of high spin operators [16, 17, 18]. The Wilson loop approach considered here is a very efficient way of computing this quantity.

\section{INTEGRAL FUNCTIONS}

Let us discuss the different kinematical regions for complex $x$. It is useful to recall the relationship of $\Gamma_{\text {cusp }}$ to IR divergences of massive form factors, such as $\gamma^{*} \rightarrow e^{+} e^{-}$, which have the same analytical structure.

$\Gamma_{\text {cusp }}$ is real in the Euclidean region $0<x<1$, and in the physical region below threshold (of creating two massive particles), which is the unit circle (i.e. for $\phi$ real). The threshold is at $x=-1$, and the physical region above threshold goes from -1 to 0 along the negative real axis. See Fig. 2 .

We find that all results can be expressed in terms of harmonic polylogarithms (HPL) [19]. These functions are generalizations of classical polylogarithms, and appear naturally in this problem. They are defined iteratively by

$$
H_{a_{1}, a_{2}, \ldots, a_{n}}(x)=\int_{0}^{x} f_{a_{1}}(t) H_{a_{2}, \ldots, a_{n}}(t) d t
$$




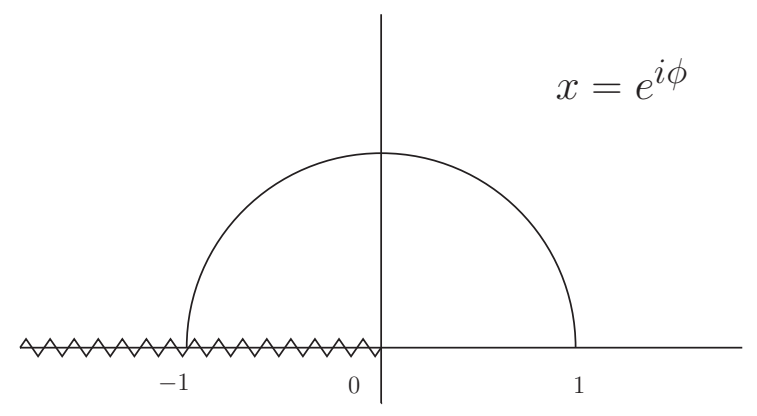

Figure 2: $\Gamma_{\text {cusp }}$ is real for $0<x<1$, and below threshold, where $x=e^{i \phi}$ is a phase. Above threshold, we have $-1<x<0$, with $x$ having an infinitesimal imaginary part. The zigzag line denotes a branch cut along the negative real axis. Recall that $\Gamma_{\text {cusp }}$ has is an inversion symmetry $x \rightarrow 1 / x$.

where the integration kernels are defined as

$$
f_{1}(x)=(1-x)^{-1}, \quad f_{0}(x)=x^{-1}, \quad f_{-1}(x)=(1+x)^{-1},
$$

The degree (or weight) one functions needed to start the recursion are defined as

$$
H_{1}(x)=-\log (1-x), \quad H_{0}(x)=\log (x), H_{-1}(x)=\log (1+x) .
$$

The subscript of $H$ is called the weight vector. A common abbreviation is to replace occurrences of $m$ zeros to the left of \pm 1 by $\pm(m+1)$. For example, $H_{0,0,1,0,-1}(x)=H_{3,-2}(x)$.

HPLs have simple properties under certain argument transformations, and one can use their algebraic properties in order to make their asymptotic behavior manifest. We refer the interested reader to ref. [19]. A very useful computer algebraic implementation has been given in ref. [20].

The perturbative results given in section 4 can be straight forwardly evaluated numerically in the region $0<x<1$. Other regions can be reached by analytical continuation, respecting the above branch cut properties. A related discussion is given in ref. [21] for the process $g g \rightarrow h$ via a massive quark loop.

\section{RESULTS UP TO THREE LOOPS}

The cusp anomalous dimension of the standard bosonic Wilson loop operator was computed in QCD at two loops in the pioneering paper [9]. This result was later simplified [22], and recomputed in $\mathscr{N}=4 \mathrm{SYM}$ for the supersymmetric loop operator defined in eq. (1.1) in refs. [23, 24]. The answer in $\mathscr{N}=4 \mathrm{SYM}$ can be written as an expansion in the "t Hooft coupling $\lambda$,

$$
\Gamma_{\text {cusp }}=\sum_{L \geq 1}\left(\frac{\lambda}{8 \pi^{2}}\right)^{L} \Gamma_{\text {cusp }}^{(L)} .
$$

To two loops it is given by,

$$
\begin{aligned}
& \Gamma_{\text {cusp }}^{(1)}=-\xi \log x, \\
& \Gamma_{\text {cusp }}^{(2)}=\frac{1}{3} \xi\left[\log x\left(\log ^{2} x+\pi^{2}\right)\right]+\frac{1}{4} \xi^{2}\left[H_{1,1,1}+2 H_{1,2}\right] .
\end{aligned}
$$


Here and in the following, the HPLs are understood to have argument $1-x^{2}$. In ref. [14], we computed the three-loop value $\Gamma_{\text {cusp }}^{(3)}$. Before outlining the method of calculation in section 6 , let us present the result. Here we write it in a very compact form, due to [25],

$$
\begin{aligned}
\Gamma_{\text {cusp }}^{(3)} & =-\frac{1}{6} \xi \log x\left(\log ^{2} x+\pi^{2}\right)^{2} \\
& -\frac{1}{2} \xi^{2}\left[3 \zeta_{3} H_{1,1}+\zeta_{2}\left(2 H_{1,2}+4 H_{2,1}+3 H_{1,1,1}\right)+2 H_{1,1,1,2}+\frac{3}{2} H_{1,2,1,1}+2 H_{2,1,1,1}+\frac{11}{4} H_{1,1,1,1,1}\right] \\
& +\frac{1}{4} \xi^{3}\left[4 H_{1,1,3}+4 H_{1,2,2}+4 H_{1,1,1,2}+2 H_{1,1,2,1}+2 H_{1,2,1,1}+3 H_{1,1,1,1,1}\right] .
\end{aligned}
$$

Let us now discuss these formulas.

It is not hard to verify the expected inversion symmetry $x \rightarrow 1 / x$, i.e. $\phi \rightarrow-\phi$, using standard relations between HPLs of related arguments [19]. Note that $\xi$ is antisymmetric under this transformation.

We see that at each loop order, we have functions of uniform degree of transcendentality (i.e. weight of harmonic polylogarithms) $(2 L-1)$.

It is remarkable that the result can be written using argument $1-x^{2}$ (or $x^{2}$ ) only. This is not the case in general for individual Feynman integrals. Moreover, we see that there are no relative signs within the coefficients of the different powers of $\xi$ in the expressions above. This property also holds at least up to $L=6$ for the $\xi^{L}$ terms at $L$ loops, which have recently been computed analytically [25].

\section{RELATION TO LIGHT-LIKE CUSP ANOMALOUS DIMENSION}

Let us use the above three-loop result to analytically verify the relation

$$
\lim _{x \rightarrow 0} \Gamma_{\text {cusp }}=-\frac{1}{2} \log x \Gamma_{\text {cusp }}^{\infty}+\mathscr{O}\left(x^{0}\right),
$$

where $\Gamma_{\text {cusp }}^{\infty}$ is the anomalous dimension of a light-like cusped Wilson loop [26]. The asymptotic limit $x \rightarrow 0$ is easy to perform on our results, since the logarithmic behavior of HPLs can always be made manifest [19]. From eqs. (4.2), (4.3) and (4.4) it is easy to verify its correct three-loop value [27]

$$
\Gamma_{\text {cusp }}^{\infty}=2\left(\frac{\lambda}{8 \pi^{2}}\right)-2 \zeta_{2}\left(\frac{\lambda}{8 \pi^{2}}\right)^{2}+11 \zeta_{4}\left(\frac{\lambda}{8 \pi^{2}}\right)^{3}+\mathscr{O}\left(\lambda^{4}\right)
$$

\section{RELATION TO MASSIVE SCATTERING AMPLITUDES IN N=4 SYM}

The velocity-dependent cusp anomalous dimension governs the infrared divergences of massive scattering amplitudes. In $\mathscr{N}=4$ super Yang-Mills, one can introduce masses by giving a vacuum expectation value to some of the scalar particles. In this way, at the planar level, one can define finite four-dimensional scattering amplitudes which have an exact dual conformal symmetry [28]. The simplest case is the four-particle scattering amplitude $M_{4}(u, v)$, which is a function of two conformally invariant variables $u$ and $v$, which in turn are defined in terms of the momenta and masses. 


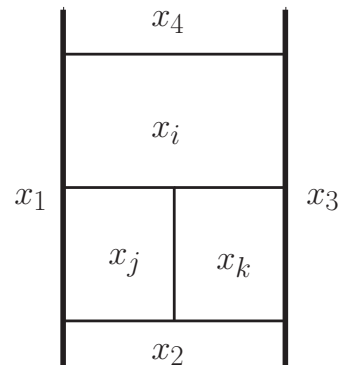

(a)

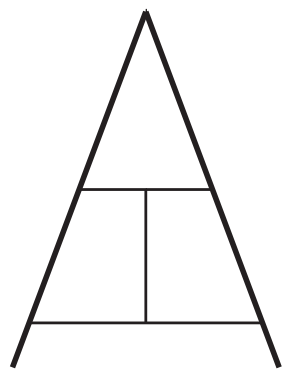

(b)

Figure 3: The form factor (or Wilson loop) integrand is obtained by taking the limit where all dual integration points, $x_{i}, x_{j}, x_{k}$ in (a), come close to the external point $x_{2}$. Here we show a three-loop integral for the four-particle amplitude (a) giving rise to contribution for the Wilson loop, shown in (b). Thick lines represent massive (or eikonal) propagators, and thin lines represent massless propagators.

It was argued in $[29,30,14]$ that the limit $u \ll 1$ of this function is determined by the cusp anomalous dimension in the following way,

$$
\lim _{u \rightarrow 0} \log M_{4}(u, v)=\log u \Gamma_{\text {cusp }}(v)+\mathscr{O}\left(u^{0}\right)
$$

The relation between $v$ and the previously used variables is $v=x /(1-x)^{2}$.

Let us explain how we used this limit to compute $\Gamma_{\text {cusp }}$ at three loops [14], starting from a known representation of the three-loop scattering amplitude [27, 29]. We observed that all known form factor and Wilson line integrals of this type could be expressed in terms of harmonic polylogarithms of argument $x$. Assuming that this holds for $\Gamma_{\text {cusp }}$ at three loops reduced the calculation to the problem of determining a number of coefficients. We used Mellin-Barnes techniques [29] to compute the asymptotic limit $x \rightarrow 0$, keeping not only powers of $\log x$, but also terms suppressed by powers of $x$. In this way, we were able to fix our ansatz completely.

We note that recently, our assumption was proven for two infinite classes of integrals, and moreover that it is possible to directly evaluate the corresponding integrals [25].

We wish to emphasize that the relation to the four-particle amplitude discussed above can also be used at the level of the integrand, where the dominant region of integration as $u \rightarrow 0$ corresponds to all dual loop momenta ${ }^{1}$ approaching one of the external dual variables, say $x_{2}^{\mu}$. In that region, one can simplify the integrand (to logarithmic accuracy) to obtain integrals of form factor type. In the same spirit, one can further approximate them in order to get a Wilson line representation. We illustrate this simplification in Fig. 3.

In summary, this means that the integrand of the Wilson line calculation can straightforwardly be obtained at higher loop orders from the corresponding integrand of massless scattering amplitudes [31, 32, 33], extended properly to the massive case [28, 29]. This should represent a useful starting point for calculations at higher loop orders, as well as for finding and proving conjectures about the structure of $\Gamma_{\text {cusp }}$, see e.g. section 8 .

\footnotetext{
${ }^{1}$ Here we are using the notion of dual (or region) variables for a planar graph.
} 


\section{EXACT RESULT FOR SMALL ANGLES}

It turns out that there is a special limit of $\Gamma_{\text {cusp }}$, namely the small angle limit $\left|\theta^{2}-\phi^{2}\right| \ll 1$, which can be computed exactly in $\phi, \lambda$ and $N$. Here we simply quote the result and refer the interested reader to [10] for more details. Specifying to the planar case $N \gg 1$, we have

$$
\Gamma_{\text {cusp }}=\left(\phi^{2}-\theta^{2}\right) \frac{1}{1-\phi^{2} / \pi^{2}} B(\tilde{\lambda})+\ldots,
$$

with $\tilde{\lambda}=\lambda\left(1-\phi^{2} / \pi^{2}\right)$, and

$$
B(\lambda)=\frac{1}{4 \pi^{2}} \frac{\sqrt{\lambda} I_{2}(\sqrt{\lambda})}{I_{1}(\sqrt{\lambda})}+\mathscr{O}\left(1 / N^{2}\right)
$$

where $I_{j}$ is the modified Bessel function.

Expanding this exact result to the third order in $\lambda$, one reproduces the coefficient of $\xi$ in eqs. (4.2), (4.3) and (4.4), respectively.

One can also expand our exact result at strong coupling. This can be compared against taking the limit $|\phi-\theta| \ll 1$ of the formulas in [24]. In both cases, we find

$$
\Gamma_{\text {cusp }}=(\theta-\phi) \frac{\sqrt{\lambda}}{2 \pi} \frac{\phi}{\sqrt{\pi^{2}-\phi^{2}}}+\ldots
$$

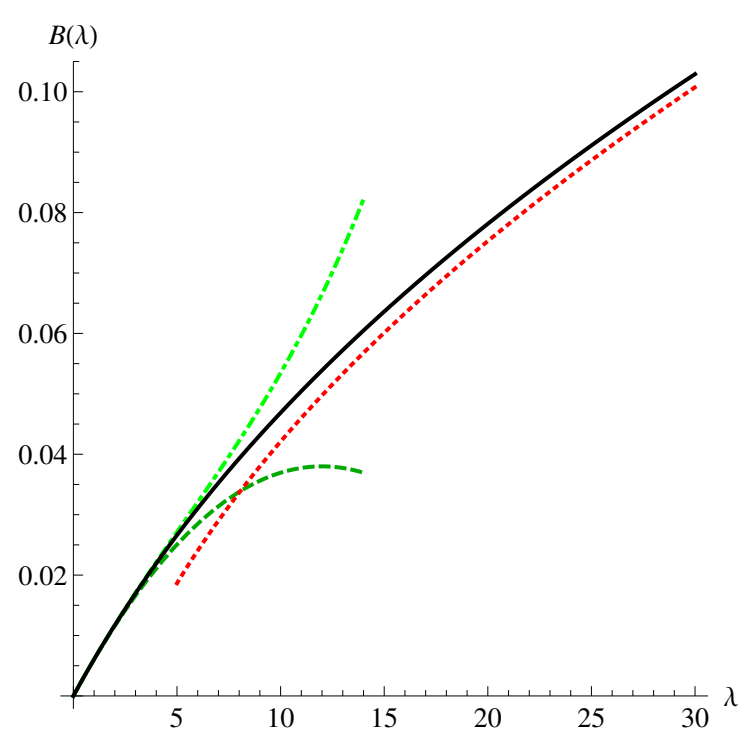

Figure 4: Bremsstrahlung function $B(\lambda)$ (solid black line) given in the main text. The dotted green lines on the left denote two-loop and three-loop approximations, respectively, and the dotted red line on the right corresponds to the first two terms of the strong coupling approximation.

For a constant coupling of the Wilson loop to scalars, i.e. $\theta=0$, eq. (7.1) becomes

$$
\Gamma_{\text {cusp }}=-\phi^{2} B(\lambda)+\mathscr{O}\left(\phi^{4}\right), \quad \theta=0 .
$$

This is related to the radiation emitted by a quark undergoing a small change in direction [10]. The "Bremsstrahlung" function $B(\lambda)$ is plotted in Fig. 4. 


\section{SCALING LIMIT}

The existence of the parameter $\theta$ allows us to define a new scaling limit. Recall that $\Gamma_{\text {cusp }}$ depends on $\cos \theta$ in a polynomial way. It is easy to see that the terms with the highest power $\cos ^{L} \theta$ at $L$ loops come from a diagram with $L$ scalar propagators ending on each Wilson line. We can isolate such terms by taking the limit [14]

$$
\lambda \rightarrow 0, \quad e^{i \theta} \rightarrow \infty, \quad \kappa=\frac{1}{4 \pi^{2}} \lambda e^{i \theta} \text { fixed }
$$

In this way, we get a non-trivial function of $\phi, \kappa$ and $N$. We see that at leading order (LO) in the limit, only ladder diagrams remain. We will denote the leading order approximation by $\Gamma_{\text {cusp }}^{\mathrm{LO}}$.

The sum of the ladder integrals satisfies a Bethe-Salpeter equation. Since we are only interested in the leading UV divergence (of the sum of the ladder integrals), we can simplify the equation to a one-dimensional Schrödinger problem,

$$
\left[-\partial_{y}^{2}-\frac{\kappa}{8} \frac{1}{\cosh y+\cos \phi}\right] \Psi(y)=-\frac{\Omega^{2}}{4} \Psi(y) .
$$

Here $y$ is a variable in an auxiliary space (related to relative positions on the two edges of the Wilson lines), and $\Gamma_{\text {cusp }}^{\mathrm{LO}}=-\Omega_{0}$, where $\Omega_{0}$ is computed for the ground state of the system.

In the case of zero angle, the potential in eq. (8.2) becomes the integrable Pöschl-Teller potential $\cosh ^{-2}(y / 2)$, and one can solve for $\Gamma_{\text {cusp }}^{\mathrm{LO}}$ in closed form. One finds [14]

$$
\Gamma_{\text {cusp }}^{\mathrm{LO}}=\frac{1-\sqrt{1+\kappa}}{2}-\frac{\phi^{2}}{16} \kappa\left(\frac{1+\sqrt{1+\kappa}}{1+\kappa+2 \sqrt{1+\kappa}}\right)+\mathscr{O}\left(\phi^{4}\right) .
$$

Moreover, for general angle, the problem can be solved perturbatively. One can show that to any order $L$, the LO answer for $\Gamma_{\text {cusp }}$ can be written in terms of harmonic polylogarithms of degree $(2 L-1)$, and an algorithm to compute the solution was given [25]. Moreover, the result was given explicitly up to six loops. This calculation also confirmed analytically the $\xi^{3}$ term at three loops that was given in equation (4.4).

There are a number of interesting properties of these results [25]. A study of the explicit results to six loops shows that in fact only a subset of harmonic polylogarithms is needed. We already mentioned in section 4 the absence of relative signs. Finally, when studying the limit $x \rightarrow 0$ discussed in section 5, one finds, at least up to six loops, that the asymptotic expansion of $\Gamma_{\text {cusp }}^{\mathrm{LO}}$ does not require multiple zeta values [34] of depth two or higher.

One can also compare the solution of the Schrödinger equation to what one obtains from taking the scaling limit of the corresponding string theory result. A priori, the answer obtained does not have to be the same, since the order of limits could be important. However, the explicit calculation showed agreement [14].

This analysis has recently been extended to the next-to-leading order (NLO) in the scaling limit [25]. (See also ref. [35] for an analysis of the special case $\phi \rightarrow \pi$.) One finds that at NLO there are two infinite classes of scalar integrals, shown in Fig. 5. They satisfy modified Bethe-Salpeter equations and can be computed algorithmically [25]. 

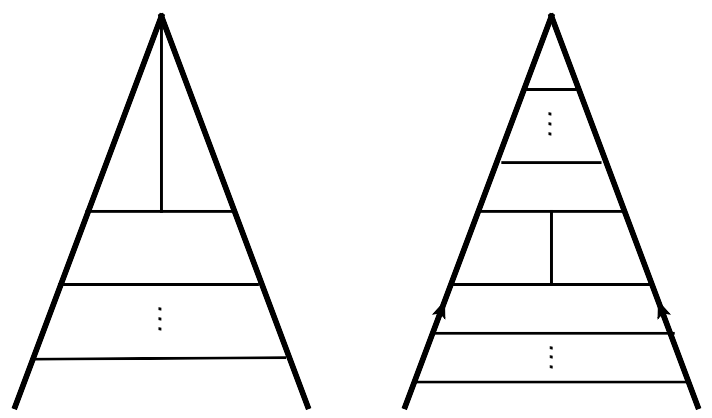

Figure 5: The two infinite classes of scalar integrals the contribute to $\Gamma_{\text {cusp }}$ at NLO in the scaling limit. discussed in the main text. The integral on the right contains a numerator factor $\left(k_{1}+k_{2}\right)^{2}$, where $k_{1}$ and $k_{2}$ are the momenta flowing along the propagators with the arrows, respectively.

\section{DISCUSSION}

We have reviewed various new results for the cusp anomalous dimension in $\mathscr{N}=4$ super Yang-Mills.

Recently, it was shown how to apply integrability techniques to this problem [36, 37, 38]. It would be interesting to reproduce the results obtained here using these methods.

While the results presented here are valid for supersymmetric gauge theories, the structures found are also be relevant for QCD. Interesting extensions of our work would be the calculation of the three-loop value of the cusp anomalous dimension in QCD, as well as the first non-planar correction, which appears for the first time at four loops.

\section{ACKNOWLEDGMENTS}

This talk is based on the publications [10, 14] with D. Correa, J. Maldacena and A. Sever, and on [25] with T. Huber. It was given at the conferences Loops and Legs in Quantum Field Theory, DESY, 2012; Scattering Amplitudes: from QCD to maximally supersymmetric Yang-Mills theory and back, ECT, 2012; Calculations for Modern and Future Colliders, CALC, 2012; The Geometry of Scattering Amplitudes, BIRS, 2012. We wish to thank the organizers for their invitation, and the participants for many interesting discussions and comments. JMH was supported in part by the Department of Energy grant DE-FG02-90ER40542.

\section{References}

[1] A. M. Polyakov, Nucl. Phys. B 164, 171 (1980).

[2] G. P. Korchemsky and A. V. Radyushkin, Phys. Lett. B 279 (1992) 359 [hep-ph/9203222].

[3] M. Neubert, Phys. Rept. 245 (1994) 259 [hep-ph/9306320].

[4] A. V. Manohar and M. B. Wise, Camb. Monogr. Part. Phys. Nucl. Phys. Cosmol. 10 (2000) 1.

[5] A. G. Grozin, Springer Tracts Mod. Phys. 201 (2004) 1.

[6] J. M. Maldacena, Phys. Rev. Lett. 80 (1998) 4859 [hep-th/9803002].

[7] S. -J. Rey and J. -T. Yee, Eur. Phys. J. C 22, 379 (2001) [hep-th/9803001]. 
[8] R. A. Brandt, F. Neri and M. -a. Sato, Phys. Rev. D 24 (1981) 879.

[9] G. P. Korchemsky and A. V. Radyushkin, Nucl. Phys. B 283 (1987) 342.

[10] D. Correa, J. Henn, J. Maldacena and A. Sever, JHEP 1206 (2012) 048 [arXiv:1202.4455 [hep-th]].

[11] B. Fiol, B. Garolera and A. Lewkowycz, JHEP 1205 (2012) 093 [arXiv:1202.5292 [hep-th]].

[12] J. K. Erickson, G. W. Semenoff, R. J. Szabo and K. Zarembo, Phys. Rev. D 61 (2000) 105006 [hep-th/9911088].

[13] A. Pineda, Phys. Rev. D 77 (2008) 021701 [arXiv:0709.2876 [hep-th]].

[14] D. Correa, J. Henn, J. Maldacena and A. Sever, JHEP 1205 (2012) 098 [arXiv:1203.1019 [hep-th]].

[15] M. Stahlhofen, arXiv:1209.2122 [hep-th].

[16] G. P. Korchemsky, Parton Distributions,” Mod. Phys. Lett. A 4 (1989) 1257.

[17] G. P. Korchemsky and G. Marchesini, Nucl. Phys. B 406 (1993) 225 [hep-ph/9210281].

[18] L. F. Alday and J. M. Maldacena, JHEP 0711 (2007) 019 [arXiv:0708.0672 [hep-th]].

[19] E. Remiddi and J. A. M. Vermaseren, Int. J. Mod. Phys. A 15 (2000) 725 [hep-ph/9905237].

[20] D. Maitre, Comput. Phys. Commun. 174 (2006) 222 [hep-ph/0507152].

[21] C. Anastasiou, S. Beerli, S. Bucherer, A. Daleo and Z. Kunszt, JHEP 0701 (2007) 082 [hep-ph/0611236].

[22] N. Kidonakis, Phys. Rev. Lett. 102 (2009) 232003 [arXiv:0903.2561 [hep-ph]].

[23] Y. Makeenko, P. Olesen and G. W. Semenoff, Nucl. Phys. B 748 (2006) 170 [hep-th/0602100].

[24] N. Drukker and V. Forini, JHEP 1106 (2011) 131 [arXiv:1105.5144 [hep-th]].

[25] J. M. Henn and T. Huber, arXiv:1207.2161 [hep-th].

[26] I. A. Korchemskaya and G. P. Korchemsky, Phys. Lett. B 287 (1992) 169.

[27] Z. Bern, L. J. Dixon and V. A. Smirnov, Phys. Rev. D 72 (2005) 085001 [arXiv:hep-th/0505205].

[28] L. F. Alday, J. M. Henn, J. Plefka and T. Schuster, JHEP 1001 (2010) 077 [arXiv:0908.0684 [hep-th]].

[29] J. M. Henn, S. G. Naculich, H. J. Schnitzer and M. Spradlin, JHEP 1004 (2010) 038 [arXiv:1001.1358 [hep-th]].

[30] J. M. Henn, Nucl. Phys. Proc. Suppl. 205-206 (2010) 193 [arXiv:1005.2902 [hep-ph]].

[31] N. Arkani-Hamed, J. L. Bourjaily, F. Cachazo, S. Caron-Huot and J. Trnka, JHEP 1101 (2011) 041 [arXiv:1008.2958 [hep-th]].

[32] J. L. Bourjaily, A. DiRe, A. Shaikh, M. Spradlin and A. Volovich, JHEP 1203 (2012) 032 [arXiv:1112.6432 [hep-th]].

[33] B. Eden, P. Heslop, G. P. Korchemsky and E. Sokatchev, Nucl. Phys. B 862 (2012) 450 [arXiv:1201.5329 [hep-th]].

[34] J. Blumlein, D. J. Broadhurst and J. A. M. Vermaseren, Comput. Phys. Commun. 181, 582 (2010) [arXiv:0907.2557 [math-ph]].

[35] D. Bykov and K. Zarembo, arXiv:1206.7117 [hep-th].

[36] D. Correa, J. Maldacena and A. Sever, JHEP 1208 (2012) 134 [arXiv:1203.1913 [hep-th]].

[37] N. Drukker, arXiv:1203.1617 [hep-th].

[38] N. Gromov and A. Sever, arXiv:1207.5489 [hep-th]. 